\title{
Inclusión educativa en relación con la cultura y la convivencia escolar*
}

\section{René Valdés-Morales}

orcid.org/00oo-0003-4242-9748

Pontificia Universidad Católica de

Valparaíso, Chile

rene.valdes@pucv.cl

\section{Verónica López}

orcid.org/0000-0001-7405-3859 Pontificia Universidad Católica de Valparaíso, Chile veronica.lopez@pucv.cl

\section{Felipe Jiménez-Vargas}

orcid.org/00oo-0002-1081-9250

Pontificia Universidad Católica de

Valparaíso, Chile

felipe.jimenez@pucv.cl

\section{Resumen}

El objetivo de este estudio es caracterizar la convivencia y la cultura escolar desde la perspectiva de la inclusión. El diseño del estudio es mixto: evaluación cuantitativa de las dimensiones convivencia escolar inclusiva y cultura inclusiva, en 129 escuelas chilenas ( $N=2868$ estudiantes, 1129 padres y 741 docentes y profesionales de la educación). Luego, seleccionamos dos casos extremos y realizamos una aproximación etnográfica. Los resultados indican que la escuela con baja convivencia escolar y baja cultura inclusiva mantenía relaciones interpersonales fragmentadas, con desorganización de roles y funciones y con un foco de interés prioritario puesto en la mejora de la convivencia. En contraste, la escuela con alta convivencia escolar y alta cultura inclusiva presentaba relaciones humanas cohesionadas, claridad de roles y funciones y su actual foco era de carácter pedagógico.

\section{Palabras clave (fuente: tesauro de la Unesco)}

Convivencia escolar; inclusión educativa; cultura escolar; etnografia; etnología; Chile.

\footnotetext{
* $\quad$ Financiamiento: PIA CONICYT CIE 160009, FONDECYT No. 1140960, FONDECYT No. 1191267 y Beca de doctorado No. 21170256 CONICYT, Chile.
}

Recepción: 23/07/2018 | Envío a pares: 11/03/2019 | Aceptación por pares: 25/03/2019 | Aprobación: 17/05/2019 


\title{
Educational Inclusion in Relation to School Culture and Coexistence*
}

\begin{abstract}
The objective of this study is to characterize school coexistence and culture from the perspective of inclusion. The study has a mixed-method design; quantitative assessment was conducted on the inclusive school coexistence and inclusive culture dimensions in 129 Chilean schools ( $N=2,868$ students, 1,129 parents and 741 teachers and education professionals). Then, we selected two extreme cases and applied an ethnographic approach. Results indicate that the school with low school coexistence and low inclusive culture maintained fragmented interpersonal relationships, with disorganized roles and functions, and emphasis placed on the improvement of school coexistence. In contrast, the school with high school coexistence and high inclusive culture showed cohesive human relationships, clarity of roles and functions, and a pedagogical focus.
\end{abstract}

\section{Keywords (Source: Unesco Thesaurus)}

School coexistence; education inclusion; school culture; ethnography; ethnology; Chile.

* $\quad$ Finance: PIA CONICYT CIE 1600og, FOnDECYT No. 1140960, FONDECYT No. 1191267, and doctorate scholarship No. 21170256 CONICYT, Chile. 


\section{Inclusão educativa em relação à cultura e à convivência escolar*}

Resumo

O objetivo deste estudo é caracterizar a convivência e a cultura escolar a partir da perspectiva da inclusão. O desenho do estudo é misto: avaliação quantitativa das dimensões convivência escolar inclusiva e cultura inclusiva em 129 escolas chilenas ( $N=2.868$ estudantes, 1.129 pais e 741 docentes e profissionais da educação). Logo, selecionamos dois casos extremos e realizamos uma aproximação etnográfica. Os resultados indicam que a escola com baixa convivência escolar e baixa cultura inclusiva mantinha relações interpessoais fragmentadas, com desorganização dos papéis e das funções, e com um foco de interesse prioritário na melhora da convivência. Em compensação, a escola com alta convivência escolar e alta cultura inclusiva apresentava relações humanas coesas, clareza de papéis e funções, e seu atual foco era de caráter pedagógico.

\section{Palavras-chave (Fonte: tesauro da Unesco)}

Coexistência escolar; inclusão educacional; cultura escolar; etnografia; etnologia; Chile.

* Financiamento: PIA CONICYT CIE 160009, FondeCYT No. 1140960, FondeCYT No. 1191267, e bolsa de doutoramento No. 21170256 CONICYT, Chile. 


\section{El desarrollo de escuelas inclusivas}

Los sistemas escolares son hoy más diversos que nunca. La amplia cobertura de la escolarización ha dejado de manifiesto pluralidades étnicas, culturales, religiosas, sexuales y/o físicas dentro de las escuelas, las cuales se encuentran potencialmente más vulnerables a sufrir situaciones de discriminación y segregación. En ese sentido, es un desafío ético y social dar respuesta a la diversidad no solo en la escuela, sino en la sociedad en su conjunto. En este desafío, la educación inclusiva asoma como la opción más auténtica para minimizar barreras y reducir la exclusión del sistema educativo. Desde esta perspectiva, la inclusión educativa se entiende como el proceso que asegura el acceso, la participación y el aprendizaje de todos los estudiantes vulnerables a ser sujetos de exclusión, no solo aquellos con discapacidad o categorizados con necesidades educativas especiales (Unesco, 2017).

A nivel internacional, durante las últimas décadas han sido variadas las iniciativas que se han llevado a cabo para transformar las escuelas, especialmente aquellas ubicadas en contextos en riesgo de exclusión. Nos referimos a las diferentes modalidades de integración escolar fundadas en los años 80 y 90 y a las actuales perspectivas de inclusión educativa. Los primeros hitos nacen con la conocida Declaración de Salamanca (1994), firmada por 300 participantes en representación de 92 países y 25 organizaciones internacionales, que afirma que las escuelas con orientación inclusiva representan el medio más eficaz para combatir las desigualdades (Unesco, 1994). A esta iniciativa le siguen otras, como el Index for Inclusion (2000), el movimiento Improving Quality Education for All (2003), la Agencia Europea para el Desarrollo de la Educación Especial (1996), así como el respaldo de organismos internacionales como la Organización de las Naciones Unidas para la Educación, la Ciencia y la Cultura (Unesco), la Organización para la Cooperación y el Desarrollo Económicos (OCDE), el Fondo de las Naciones Unidas para la Infancia (Unicef), entre otros.
Ahora bien, educación inclusiva y escuela inclusiva no son sinónimos (Valcarce, 2011). Aquel es un concepto más amplio que va más allá de la educación formal o de las instituciones escolares, mientras que escuela inclusiva remite al espacio formal donde se prescribe la oferta educativa a la diversidad, aspirando a la plena escolarización: acceso, permanencia, promoción, participación y aprendizaje (Unesco, 2017). Esta investigación se desarrolla en el marco de las escuelas inclusivas. La literatura ha identificado algunas de sus características claves (Stainback y Stainback, 1999; Booth y Ainscow, 2000; Blanco, 2006; Valcarce, 2011): a) conciben la diversidad con base en un modelo social, es decir, sostienen que las diferencias individuales dependen de la interacción entre factores intrínsecos y extrínsecos; $b$ ) diseñan e implementan estrategias de enseñanzaaprendizaje adaptadas y diversificadas; c) cuentan con un liderazgo escolar que combina participación, colaboración, colegialidad y coordinación y poseen un proyecto educativo que hace suyo el discurso de la valoración de la diversidad; d) mantienen una fuerte relación entre la escuela y su entorno; e) cuentan con aulas inclusivas que parten de la filosofía de que todos los estudiantes pertenecen al grupo y que todos pueden aprender en la escuela regular; $y \mathrm{f}$ ) se reconocen prácticas propias de una convivencia escolar inclusiva y una cultura escolar que celebra la diversidad como eje nuclear para el conjunto de prácticas de la institución. El foco del presente estudio es este último aspecto: la relación entre convivencia escolar, cultura escolar y escuela inclusiva.

\section{Convivencia escolar y cultura escolar inclusiva: aportes para una escuela inclusiva}

Desarrollar escuelas inclusivas no es tarea fácil: los obstáculos son múltiples y no solo están presentes dentro de la escuela, sino también fuera de ella, como los que imponen las políticas educativas, la formación del profesorado, los recursos disponibles, entre otros. En cuanto a las barreras existentes dentro de las instituciones, autores como Booth y 
Ainscow (2000), Sekkel, Zabelatto y Brandao (2010), López (2012) y Agell, Sala y Torrent (2014) concuerdan en la presencia de tres dimensiones, aunque con distintos nombres, para agrupar las barreras. Estas dimensiones se sitúan en los planos: 1) de la cultura escolar, incluyendo los valores de la comunidad, sus creencias y actitudes, 2) de los procesos de liderazgo, coordinación y funcionamiento del centro, y 3 ) de las prácticas de aula.

Si bien el plano de la cultura escolar ocupó bastante atención en los años 9o, luego su atención fue decayendo (Booth y Ainscow, 2000). Autores como Dorczak (2013), Tirado y Conde (2015), Ashikali y Groeneveld (2015), Booth y Ainscow (2015) y Moliner, Sales y Escobedo (2016) exponen argumentos a favor del estudio de las culturas inclusivas. En primer lugar, la cultura inclusiva es un elemento de identificación y comprensión de la inclusión: se puede reconocer una escuela inclusiva por la cultura que esta tenga. En segundo lugar, la cultura emerge como motor de partida en el desarrollo de una escuela inclusiva: no se puede pensar la transformación de una institución sin considerar la modificabilidad del ámbito cultural. En tercer lugar, y según el Index for Inclusion en todas sus ediciones (Booth y Ainscow, 2000, 2002, 2004 2006, 2011, 2015), la cultura inclusiva es una de las tres dimensiones claves para el cambio escolar,junto a las políticas y las prácticas. Por último, según Rodríguez y Ossa (2014), la cultura inclusiva ejerce una influencia de éxito o fracaso en una escuela que busca atender a la diversidad.

Una cultura escolar inclusiva, según Ainscow y Booth (2015), se relaciona con la creación de una comunidad escolar segura, acogedora, colaboradora y estimulante, en la que cada cual es valorado, base fundamental para que todo estudiante tenga mayores logros. Se refiere, asimismo, al desarrollo de valores inclusivos, compartidos por todo el personal de la escuela, los estudiantes, los miembros del consejo escolar y las familias, que se transmiten a todos los nuevos miembros de la comunidad escolar.
Las definiciones anteriores guardan estrecha relación con lo que la literatura sobre clima y convivencia escolar ha venido definiendo como un ambiente propicio para el aprendizaje: aquel donde prima la seguridad, la acogida, la colaboración y la estimulación y valoración de todos los estudiantes (Berg y Cornell, 2016; Berkowitz, Moore, Astor y Benbenishty, 2017; Bradshaw, Waasdorp, Debnam, y Johnson, 2014; Eliot, Cornell, Gregory y Fan, 2010; Thapa, Cohen, Guffey y Higgins-D’Alessandro, 2013; Wang y Degol, 2016).

Así, en este estudio asumimos que cultura inclusiva y convivencia escolar tienen mucho en común, sobre todo cuando se trata de una convivencia escolar democrática e inclusiva (López, 2014), y buscamos identificar y articular dicha relación con base en evidencia empírica

Respecto de la literatura sobre clima y convivencia escolar, cabe señalar que la convivencia escolar, en su variante anglosajona de clima escolar (school climate) se refiere a la calidad y el carácter de la vida escolar, y se basa en los patrones de las experiencias de la vida escolar de las personas, de manera tal que se reflejan las normas, los objetivos, los valores, las relaciones interpersonales, las prácticas de enseñanza, aprendizaje y liderazgo, y las estructuras organizativas que conforman la vida escolar (Cohen, McCabe, Michelli y Pickeral, 2009). Esto remite en consecuencia al carácter e identidad de la escuela.

No obstante, en América Latina, Morales y López (2019) han identificado distintas lógicas que subyacen a los intereses y supuestos respecto de los cuales se considera necesario mejorar la convivencia escolar. Una de estas perspectivas es la reconstrucción y/o fortalecimiento de la democracia. Desde aquí, se asume que la convivencia escolar es importante, pues la escuela es un espacio idóneo para aprender y preparar la vida en democracia (Hirmas y Eroles, 2008). Las estrategias y prácticas escolares asociadas a la perspectiva de convivencia democrática guardan estrecha relación con la formación 
ciudadana, por cuanto exigen el desarrollo de competencias de formación para la vida en común. En México, Fierro (2013) y Chaparro, Caso, Fierro y Díaz (2015) han desarrollado el concepto de convivencia escolar democrática e inclusiva, definiéndola como el reconocimiento de la dignidad de todas las personas partiendo de una valoración de sus características de género, etnia, religión, cultura, grupo social y capacidades, entre otras. Al mismo tiempo, que sea democrática se refiere a la participación y corresponsabilidad en la generación y seguimiento de los acuerdos que regulan la vida en común, así como en el manejo de las diferencias y conflictos. Tras diseñar un cuestionario que evalúa prácticas de convivencia escolar democrática, inclusiva y pacífica, y luego de validarlo en México (Chaparro et al., 2015) y en Chile (Valdés, López y Chaparro, 2018), se ha constatado que las dimensiones teóricas de convivencia democrática, inclusiva y pacífica tienen asidero psicométrico, es decir, que los ítems teóricamente relacionados efectivamente se agrupan psicométricamente.

A nivel internacional, se reconoce que el clima escolar y la convivencia escolar son un medio tanto como un fin en sí mismo (López, 2014). Un medio, pues los ambientes de convivencia seguros, con normas claras y justas, así como participativos, potencian y facilitan el logro de aprendizajes escolares medidos según el rendimiento en pruebas estandarizadas (López, Ascorra, Bilbao, Morales, Moya y Oyanedel, 2012). Existe evidencia internacional que demuestra el efecto compensatorio del clima escolar sobre el rendimiento académico, por sobre lo que explica el nivel socioeconómico de las familias de los estudiantes (Berkowitz et al., 2017; Unesco, 2008; 2016). Un fin, porque la escuela debería ser un espacio donde niños y niñas construyan aprendizajes académicos y socioemocionales y aprendan a convivir de manera democrática, hasta convertirse en los protagonistas de sociedades más inclusivas, justas y participativas (Hirmas y Eroles, 2008; López, Montecinos, Rodríguez, Calderón y Contreras, 2011; Unesco, 2013). Vivir en espacios libres de violencia y aprender a vivir en sociedad se entienden, desde esta perspectiva, como un derecho y un fin por sí solos. Como señala Cohen (2006), la educación social, emocional, ética y académica es un derecho humano que se debe garantizar a todos los estudiantes, por lo que ignorarlo es una forma de injusticia social (Watts y Erevelles, 2004). Por otra parte, el aprendizaje declarativo y la experiencia procedimental de convivir democráticamente en la escuela y participar activamente en la construcción de una comunidad escolar es importante para escuela y para el bienestar psicológico y social de los estudiantes, de sus profesores y de los demás adultos de la escuela (Berg y Cornell, 2016; Bilbao, 2014; Páez y Martin-Beristain, 2011).

En este estudio planteamos que la convivencia escolar podría ser no solo un fin en sí mismo, sino también un medio o condición para la formación y el fortalecimiento de escuelas inclusivas. Si el fin es la educación inclusiva, nuestro argumento es que las políticas y prácticas locales en la escuela requieren del desarrollo de políticas y prácticas de gestión de la convivencia escolar (Bellei, Valenzuela, Vanni y Contreras, 2014; López y Carrasco, 2015; Mena, Becerra y Castro, 2011) que están estrechamente relacionadas con el desarrollo de culturas inclusivas (Anderson, 2010; Astor, Benbenishty y Estrada, 2009). Sobre la base de estos planteamientos y de los argumentos anteriormente expuestos, en esta investigación nos propusimos como objetivo general caracterizar y comprender la convivencia y la cultura a la luz de la inclusión escolar. Como objetivos específicos: 1) evaluamos cuantitativamente las dimensiones de convivencia escolar y cultura inclusiva y 2 ) profundizamos en cómo operan ambas dimensiones en la práctica educativa, realizando una aproximación etnográfica en dos escuelas identificadas con alto y bajo nivel de convivencia escolar y cultura inclusiva.

\section{Método}

Diseño: Mediante una metodología mixta secuencial (Onwuegbuzie y Leech, 2005), describimos cuantitativamente los constructos de "convivencia 
escolar inclusiva" en estudiantes y de "cultura escolar inclusiva" en padres y profesionales de 129 escuelas chilenas. Luego, mediante un abordaje cualitativo, realizamos una inmersión etnográfica de dos meses con el propósito de tener una mirada comprensiva más profunda y situada sobre los constructos antes mencionados. Tener una mirada comprensiva nos ayuda a develar las prácticas que están en la base de los datos cuantitativos. Por este mismo motivo, es necesaria la inmersión etnográfica, pues permite develar las manifestaciones socioculturales que existen dentro del espacio escolar (Díaz, 2013).

Participantes: En la fase cuantitativa participaron 2.868 estudiantes de octavo año básico, 1.129 padres y 741 profesionales de 129 escuelas de tres regiones del país, entre las cuales hay municipales, particulares subvencionadas y particulares'. Todas son urbanas. En la fase cualitativa participaron dos escuelas seleccionadas que cumplieron con la condición de "casos extremos" para realizar una inmersión etnográfica. El muestreo por casos extremos focaliza la atención en aquellos casos ricos en información y que, por tanto, permiten analizar condiciones inusuales y comprender aspectos ocultos (Quintana, 2006).

INSTRUMENTO: El instrumento destinado a estudiantes fue una adaptación de un instrumento mexicano (Caso, Chaparro y Díaz, 2013; Valdés, López y Chaparro, 2018) que evaluaba prácticas de convivencia inclusiva, democrática y pacífica. Se consideró la dimensión Convivencia Inclusiva (alfa de Cronbach = o,88) como criterio para determinar la presencia de una cultura de la inclusión según la perspectiva de estudiantes. Para su adaptación se realizó un análisis léxico de los indicadores y un análisis factorial de tipo exploratorio y confirmatorio. Los resultados mostraron una estructura factorial semejante a la versión del cuestionario original, además de apropiados

$1 \quad$ Las escuelas municipales son aquellas que reciben total subvención del estado. Las que son particulares subvencionadas reciben aportes del estado y aportes de privados. Y las escuelas particulares pagadas reciben aporte total de privados y de familias. niveles de consistencia interna, con índices alfa de Cronbach mayores de 0,70 en todas las dimensiones (Valdés, López y Chaparro, 2018). El instrumento usa la escala de Likert de 1 a 5 ( 1 = nunca, 2 = algunas veces, 3 = regularmente, 4 = casi siempre, $5=$ siempre) y la consigna fue "en esta escuela...". A esta consigna se le suma un listado de prácticas concretas.

Para el caso del instrumento destinado a padres y profesionales se construyó y validó uno que evaluara la convivencia inclusiva desde la perspectiva de las prácticas y ofreciera múltiples dimensiones (López y Valdés, 2018). Una de las dimensiones evaluó el constructo "cultura inclusiva" bajo la perspectiva de Booth y Ainscow (2015) y contó con ochos ítems que guardaban relación con los indicadores que sugiere el índice de inclusión para indagar en culturas escolares inclusivas. Como la versión para adultos estaba destinada a padres y profesionales, hubo diferencias entre cantidad de ítems y léxico ocupado entre algunas dimensiones e indicadores. Esto implicó presentar los resultados como dos versiones diferentes del mismo instrumento. Los resultados de la validación psicométrica indican que los niveles de consistencia interna son adecuados, con alfas de Cronbach de o,88 para el caso de profesionales y de o,91 para el caso padres y apoderados (López y Valdés, 2018). Como instrumento, la escala de Lickert mide las respuestas de 1 a 5 ( $1=$ nunca, 2 = algunas veces, $3=$ regularmente, 4 = casi siempre, 5 = siempre) y la consigna también fue "en esta escuela".

Los datos de la fase cuantitativa fueron producidos en el período 2015-2016 con una muestra de 129 escuelas. Una vez recogidos los datos cuantitativos, nos planteamos comprender ambas dimensiones en la práctica escolar realizando una aproximación etnográfica. Para ello, utilizamos dos criterios para seleccionar los casos extremos: que fuesen escuelas de la región de Valparaíso (65) y que todos los participantes (estudiantes, padres y profesionales) concordaran en la frecuencia de prácticas de convivencia escolar y cultura inclusiva de las 65 escuelas participantes. La escuela A presenta alta convivencia esco- 
lar según estudiantes y alta cultura inclusiva según padres y profesionales. La escuela B presenta baja convivencia escolar según estudiantes y baja cultura inclusiva según padres y profesionales. Ambas escuelas seleccionadas pertenecen a la zona norte de la región de Valparaíso. La escuela A es una escuela particular subvencionada gratuita y sin selección escolar. La escuela B es una escuela pública municipal. Ambas instituciones tienen a la inclusión escolar como uno de sus propósitos institucionales.

TÉCNICAS DE PRODUCCIÓN Y ANÁlISIS: Para la fase cuantitativa se realizó un análisis descriptivo de la frecuencia de las prácticas asociadas a las dimensiones de convivencia inclusiva, para el caso de estudiantes, y de cultura inclusiva, para el de profesionales. En la fase cualitativa utilizamos como técnicas de producción el diario de campo y entrevistas activo-reflexivas (Holstein y Gubriun, 1995), las cuales incentivan la reflexión y la participación activa en la conversación, necesaria para este estudio, ya que permite profundizar en temas claves de interés, en este caso, en las dimensiones evaluadas en la fase cuantitativa. Por tanto, no hay preguntas específicas, sino tópicos que permiten una mayor y mejor comprensión de la convivencia escolar y cultura inclusiva.

Se observaron los espacios formales de la escuela (sala de clases, reuniones de equipos directivos, reuniones de coordinación, etc.) y los espacios informales (recreos, hora del café de profesores, espacios de entrada y salida de la escuela, etc.). En el caso de las entrevistas, estas se realizaron a estudiantes, profesores, familias, profesionales de apoyo y miembros del equipo directivo. Los tópicos de las entrevistas surgieron de los indicadores consultados en la fase cuantitativa y de los temas registrados en las observaciones de campo. Por tanto, en las observaciones y en las entrevistas se buscó, en una primera fase, precisar la información del instrumento cuantitativo y luego, en una segunda fase, reflexionar activamente respecto de los temas que emergieron como resultado preliminar de la fase cualitativa y que serían relevantes para la comprensión de los constructos evaluados.

Para analizar los datos producidos, utilizamos el análisis de contenido temático (Vázquez, 1994), con categorías prestablecidas, que fueron los temas asociados a las dimensiones evaluadas, además de categorías emergentes. Este análisis contempló los siguientes momentos: a) lectura de los datos; b) codificación de citas prestablecidas y emergentes; c) reagrupamiento de citas en categorías; d) agrupamiento y selección de categorías finales; e) análisis e interpretación de resultados.

Si bien del análisis emergieron múltiples categorías, acá presentamos las categorías emergentes más densas desde el punto de vista de la codificación. Estas fueron: 1) calidad de las relaciones interpersonales, 2) organización de roles y funciones y 3) foco de la escuela. Por tanto, el análisis datos se divide en tres partes: caracterización de los datos cuantitativos, profundización cualitativa de las dimensiones evaluadas y comprensión de las categorías emergentes.

Aspectos Étıcos: Tanto para la fase cuantitativa como para cualitativa se solicitaron los asentimientos informados de estudiantes y consentimientos informados de padres y profesionales. Una vez recogidos y analizados los datos, el equipo investigador a cargo del estudio hizo llegar a las escuelas un informe de devolución con los resultados más relevantes de la investigación.

\section{Resultados}

Organizamos los resultados en dos partes. En la primera, presentamos los correspondientes a las dimensiones de "convivencia escolar inclusiva" para estudiantes y de "cultura escolar inclusiva" para apoderados y profesionales de las 129 escuelas del estudio. De la misma forma, presentamos los descriptivos para ambas escuelas del estudio. En la segunda parte, narramos la experiencia etnográfica en las dos escuelas participantes a través de una 
primera descripción de estas, en función de la profundización cualitativa de las dimensiones evaluadas y de las tres categorías emergentes antes señaladas: 1) relaciones interpersonales, 2) organización de roles y funciones y 3 ) foco de la escuela. La fase etnográfica la relatamos en primera persona plural con el propósito de mantener un relato fiel a la etnografía en escuelas (Assaél, Acuña, Contreras y Santa Cruz, 2018; Díaz, 2013).

\section{Fase cuantitativa}

En cuanto a los estadísticos descriptivos, en todos los ítems de las dimensiones analizadas, tanto para estudiantes como para padres y profesionales, se utilizaron todas las opciones de respuesta. Los valores de asimetría y curtosis son adecuados. Se observa una media y una desviación estándar estables para todos los ítems.

En relación con la dimensión de Convivencia Escolar Inclusiva reportada por estudiantes, las prácticas que presentan menor frecuencia según ellos son las que aparecen en los números 3, 5 y 7 , los cuales no sobrepasan el 50\% de la opción de respuesta "casi siempre o siempre" y hacen referencia a la capacidad de motivar de los profesores, al apoyo diferenciado en aula y a la disponibilidad del director para atender a los estudiantes, respectivamente. Las prácticas de mayor frecuencia son los números 2 y 8, que alcanzan el 70\% de respuesta en la opción "casi siempre o siempre" y guardan relación con la apertura de la escuela a la atención de padres, con reforzamiento positivo por los docentes. El resto de los items demuestran acciones que se dan regularmente en las escuelas (ver Tabla 1 y Figura 1).

Según los padres encuestados respecto de la dimensión Cultura Inclusiva, todas las prácticas tienen una frecuencia sobre el $50 \%$ y están en la opción "casi siempre o siempre". Las acciones que aparecen sobre el $70 \%$ son los items 2 y 4 , en las cuales los padres mencionan que confían y se sienten orgullos de la escuela donde están matriculados sus hijos y/o pupilos.
En relación con la dimensión Cultura Inclusiva para profesionales, las acciones menos frecuentes son aquellas referidas en los reactivos 3, 4y 8, los cuales refieren a la presencia de un proceso de inducción, a la buena comunicación entre pares y a la presencian de tradiciones. La acción más frecuente es la número 2, que se refiere al orgullo que sienten los profesionales de pertenecer a la institución donde trabaja.

Como podemos observar en las Tablas 1 y 2 , las escuelas A y B difieren entre sí en las dimensiones evaluadas. Mientras que la escuela A reporta mayor frecuencia de prácticas asociadas a una convivencia escolar inclusiva $(M=4,4)$, cultura escolar inclusiva en padres $(M=4,2)$ y cultura escolar inclusiva en profesionales $(M=4,1)$, la escuela $B$ reporta menos acciones en las dimensiones de convivencia escolar inclusiva $(M=3,6)$, cultura escolar inclusiva en padres $(M=3,5)$ y cultura escolar inclusiva en profesionales $(M=3,6)$.

En cuanto a la dimensión Convivencia Escolar Inclusiva, los indicadores que más difieren entre ambas escuelas son las relacionadas con la entrega de apoyos por parte del director de escuela. En la dimensión de Cultura Inclusiva para padres, los indicadores que más difieren en la frecuencia son aquellos relacionados con la confianza y la buena comunicación entre actores. Finalmente, desde la perspectiva de profesionales y en relación con la dimensión Cultura Inclusiva, también la confianza y la buena comunicación aparecen como elementos diferenciadores entre ambas escuelas según el instrumento aplicado.

\section{Fase cualitativa}

Como mencionamos anteriormente, mediante un muestreo de casos extremos escogimos dos escuelas. El muestreo se explica por la diferencia entre ambos centros educativos según los constructos Convivencia Escolar Inclusiva en estudiantes y Cultura Escolar Inclusiva en padres y profesionales, descritos anteriormente. 
Tabla 1. Descriptivos dimensión Convivencia Escolar Inclusiva según estudiantes

\begin{tabular}{|c|c|c|c|c|c|c|}
\hline \multirow[t]{2}{*}{ Reactivos } & \multirow{2}{*}{$\begin{array}{c}\text { Media } \\
(\mathrm{N}=\mathbf{2 8 6 8})\end{array}$} & \multirow[t]{2}{*}{ DT } & \multicolumn{2}{|c|}{$\begin{array}{l}\text { Escuela } A \\
(N=13)\end{array}$} & \multicolumn{2}{|c|}{$\begin{array}{l}\text { Escuela B } \\
(\mathbf{N}=23)\end{array}$} \\
\hline & & & $\mathbf{M}$ & DT & $\mathbf{M}$ & DT \\
\hline $\begin{array}{l}\text { 1. Los profesores(as) de mi escuela nos ayudan a ver lo que } \\
\text { cada uno puede aportar al grupo }\end{array}$ & 3,62 & 1,12 & 4,6 & 0.65 & 4,3 & 0,76 \\
\hline $\begin{array}{l}\text { 2. En mi escuela se atiende a los padres y apoderados que } \\
\text { solicitan atención especial para sus hijos }\end{array}$ & 4,14 & 1,04 & 4,6 & 0,75 & 4,1 & 1,11 \\
\hline $\begin{array}{l}\text { 3. En esta escuela los profesores(as) nos motivan para } \\
\text { conversar acerca de nuestras costumbres y tradiciones }\end{array}$ & 3,10 & 1,26 & 4,0 & 1,56 & 3,5 & 1,37 \\
\hline $\begin{array}{l}\text { 4. En mi escuela el director(a) apoya a los estudiantes con } \\
\text { problemas de conducta }\end{array}$ & 3,65 & 1,29 & 4,6 & 0,86 & 3,0 & 1,50 \\
\hline $\begin{array}{l}\text { 5. En mi escuela los profesores(as) toman tiempo de la clase } \\
\text { para apoyar a los compañeros(as) que van más atrasados }\end{array}$ & 3,29 & 1,30 & 4,0 & 1,25 & 3,5 & 1,27 \\
\hline $\begin{array}{l}\text { 6. En mi escuela los profesores(as) nos ayudan a integrarnos } \\
\text { como grupo }\end{array}$ & 3,69 & 1,19 & 4,6 & 0.96 & 3,5 & 1,20 \\
\hline $\begin{array}{l}\text { 7. En esta escuela, cuando los estudiantes tenemos problemas } \\
\text { con algún profesor(a), nuestro director(a) nos atiende }\end{array}$ & 3,31 & 1,35 & 4,8 & 0,60 & 2,5 & 1,64 \\
\hline $\begin{array}{l}\text { 8. En esta escuela los profesores(as) nos felicitan cuando } \\
\text { hacemos un buen trabajo }\end{array}$ & 3,96 & 1,17 & 4,6 & 0,63 & 4,1 & 1,14 \\
\hline $\begin{array}{l}\text { 9. En mi escuela los profesores(as) nos felicitan por nuestro } \\
\text { esfuerzo, aunque las cosas no nos salgan bien }\end{array}$ & 3,47 & 1,30 & 4,5 & 1,24 & 3,5 & 1,27 \\
\hline $\begin{array}{l}\text { 10. En mi escuela hay personas capacitadas para apoyar a } \\
\text { estudiantes con bajo rendimiento }\end{array}$ & 3,98 & 1,21 & 4,8 & 0,57 & 4,4 & 1,07 \\
\hline $\begin{array}{l}\text { 11. En esta escuela padres y apoderados y profesores(as) se } \\
\text { ponen de acuerdo para apoyarnos cuando tenemos bajas } \\
\text { calificaciones }\end{array}$ & 3,62 & 1,26 & 4,4 & 1,08 & 3,5 & 1,34 \\
\hline $\begin{array}{l}\text { 12. En esta escuela, cuando tenemos problemas de conducta, } \\
\text { se pide a los padres y apoderados que nos apoyen }\end{array}$ & 3,89 & 1,20 & 4,4 & 1,24 & 4,0 & 1,42 \\
\hline Total (promedio) & 3,64 & 1,22 & 4,4 & 0,94 & 3,6 & 1,2 \\
\hline
\end{tabular}

Fuente: elaboración propia. 


\section{Figura 1. Análisis de frecuencia según opciones de respuesta de la dimensión Convivencia Escolar Inclusiva para estudiantes}

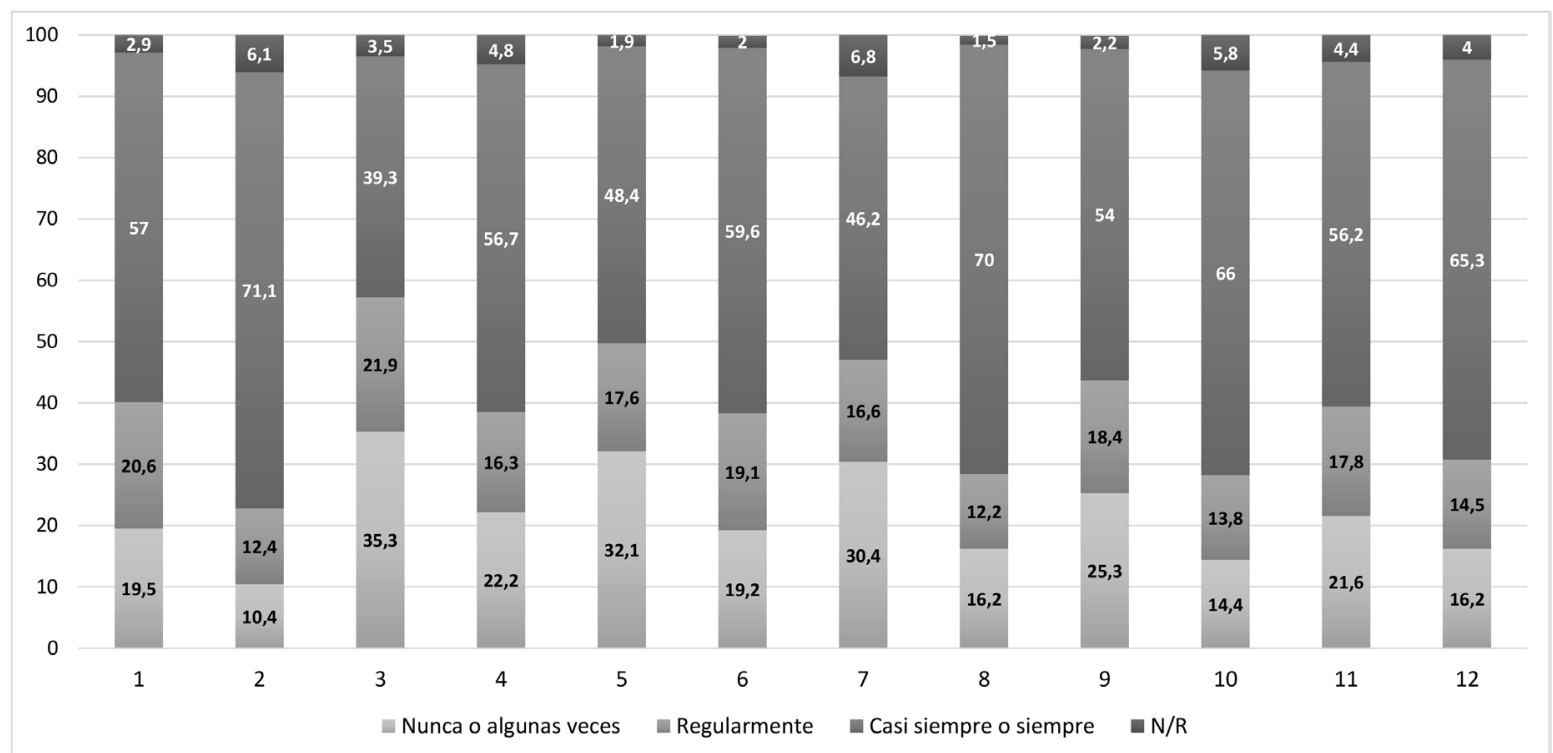

Fuente: elaboración propia.

\section{CONVIVENCIA ESCOLAR Y CULTURA INCLUSIVA:}

En relación con la comprensión cualitativa de las dimensiones evaluadas en la fase cuantitativa, en la escuela A las entrevistas realizadas a los diversos actores de la escuela concuerdan en la presencia manifiesta de una cultura inclusiva y de una buena convivencia escolar en la escuela. En relación con la dimensión Convivencia Escolar Inclusiva, los estudiantes consideran que hay profesionales idóneos en la escuela, que sus padres y apoderados reciben atención especial cuando lo necesitan y que la directora de la escuela y los profesores son claros recursos de apoyo en la mejora de la convivencia y en la integración entre compañeros. De la misma manera, destacan el ambiente familiar de la escuela: "A mí lo que más me gusta de este colegio es el ambiente, porque yo veo que los profesores y alumnos son amigos, porque varios que se van siempre vuelven al mes después; los de octavo de antes siempre vuelven

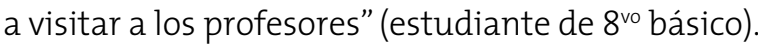

Según los profesionales, estos concuerdan en la idea de que existe ambiente familiar dentro de la escuela y perciben que los estudiantes están contentos y las familias satisfechas. De la misma manera, consideran que existe colaboración entre los distintos estamentos de la escuela. En cuanto a los padres y apoderados, para la mayoría existe ambiente familiar, hay confianza y buena comunicación entre los diversos estamentos, y las tradiciones dentro de la escuela los hacen sentir a todos partícipes de la vida escolar. La mayoría de los participantes considera que los estudiantes están contentos y las familias satisfechas.

Es que acá es como una familia. Siempre decimos eso, aunque pueda sonar asi como "ah le está poniendo", no, es verdad, acá aunque no hayas hablado con alguien y no lo conozcas, uno igual lo siente, pucha, igual. Ya si es del colegio hay que cooperar, o de repente los niños me dicen 'mamá, sabes que le pasó tal cosa al niño', [entonces] uno se preocupa, lo conversa con la mamá, con la tía, dice 'no [se preocupe], estamos haciendo esto, esto otro', y esa parte igual es buena; entonces somos como así. (Apoderada, escuela A) 
Tabla 2. Descriptivo dimensión Cultura Inclusiva según padres

\begin{tabular}{|c|c|c|c|c|c|c|}
\hline \multirow{2}{*}{ Reactivos } & \multirow{2}{*}{$\begin{array}{l}\text { Media } \\
(\mathrm{N}=1129)\end{array}$} & \multirow{2}{*}{ DT } & \multicolumn{2}{|c|}{$\begin{array}{l}\text { Escuela } A \\
(\mathrm{~N}=14)\end{array}$} & \multicolumn{2}{|c|}{$\begin{array}{l}\text { Escuela B } \\
(\mathrm{N}=\mathbf{2 0})\end{array}$} \\
\hline & & & $M$ & DT & $M$ & DT \\
\hline 1. Existe ambiente familiar y acogedor & 3,87 & 1,13 & 4,1 & 0,66 & 3,6 & 1,3 \\
\hline $\begin{array}{l}\text { 2. Nos sentimos orgullosos de participar de esta comunidad } \\
\text { escolar }\end{array}$ & 4,02 & 1,07 & 4,1 & 1,16 & 3,5 & 1,39 \\
\hline $\begin{array}{l}\text { 3. Existe confianza y buena comunicación entre todos los actores } \\
\text { de la comunidad escolar }\end{array}$ & 3,82 & 1,07 & 4,5 & 0,51 & 3,3 & 1,34 \\
\hline 4. Los padres y apoderados confían en la escuela & 4,06 & 1,01 & 4,5 & 0,65 & 3,9 & 1,14 \\
\hline 5. Existe una comunicación fluida con los padres y apoderados & 3,83 & 1,07 & 4,3 & 0,74 & 3,1 & 1,39 \\
\hline $\begin{array}{l}\text { 6. Percibimos a los estudiantes contentos y a las familias } \\
\text { satisfechas }\end{array}$ & 3,76 & 1,01 & 4 & 0,55 & 3,6 & 0,99 \\
\hline 7. Hay muchas tradiciones que nos hacen sentir parte de ella & 3,75 & 1,15 & 4 & 0,78 & 3,6 & 1,34 \\
\hline Total (promedio) & 3,87 & 0,86 & 4,2 & 0,72 & 3,5 & 1,2 \\
\hline
\end{tabular}

Fuente: elaboración propia.

Figura 2. Análisis descriptivo según opciones de respuesta de la dimensión Cultura Escolar Inclusiva para padres $y$ apoderados

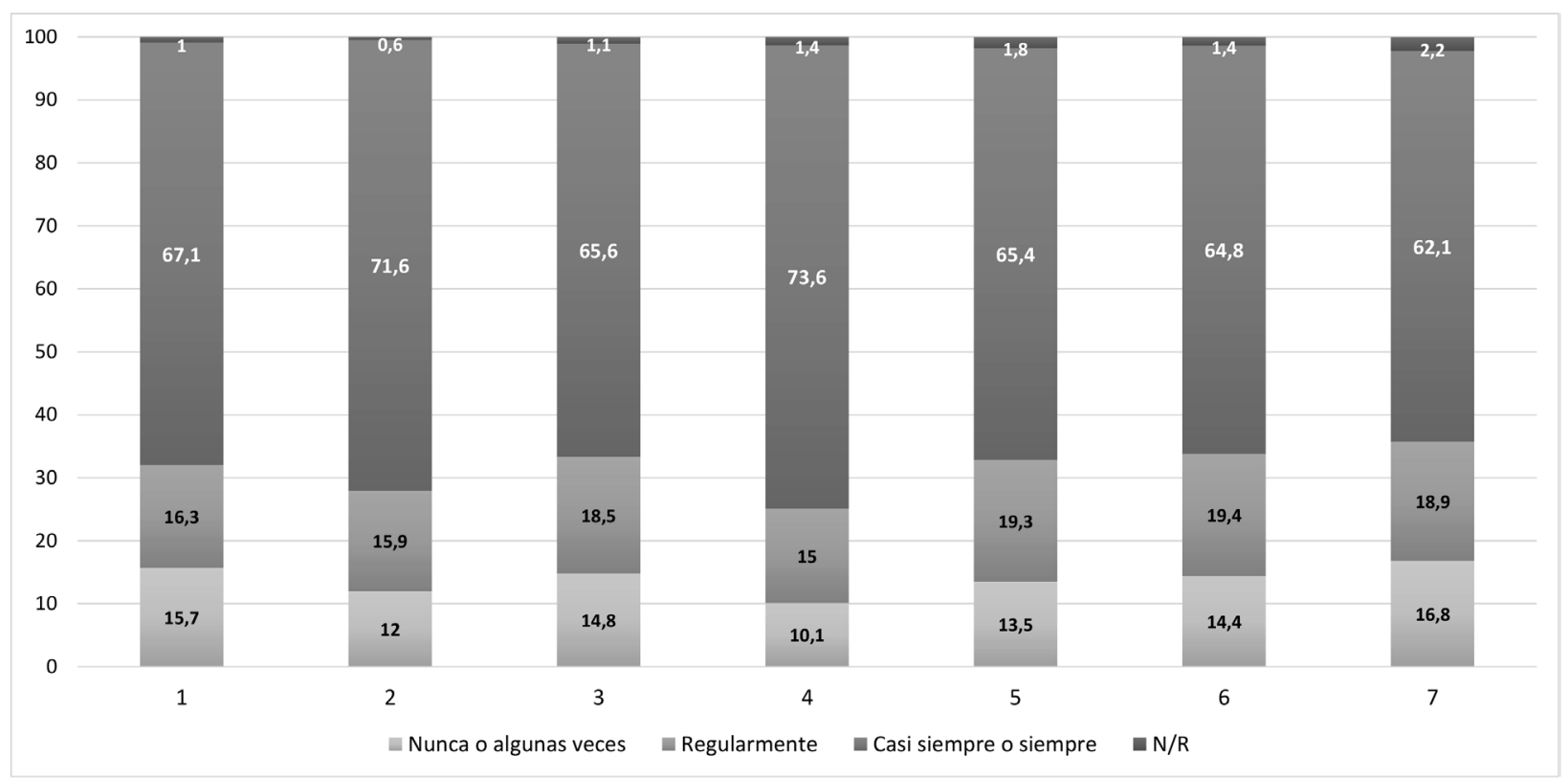

Fuente: elaboración propia. 
Tabla 3. Descriptivo dimensión Cultura Inclusiva según profesionales

\begin{tabular}{|c|c|c|c|c|c|c|}
\hline \multirow[t]{2}{*}{ Reactivos } & \multirow{2}{*}{$\begin{array}{c}\text { Media } \\
(N=741)\end{array}$} & \multirow[t]{2}{*}{ DT } & \multicolumn{2}{|c|}{$\begin{array}{c}\text { Escuela A } \\
(\mathbf{N}=9)\end{array}$} & \multicolumn{2}{|c|}{$\begin{array}{c}\text { Escuela B } \\
(\mathrm{N}=9)\end{array}$} \\
\hline & & & M & DT & M & DT \\
\hline 1. Existe un ambiente familiar & 3,80 & 1,06 & 4,4 & 1,01 & 3,4 & 1,00 \\
\hline 2. Nos sentimos orgullosos de trabajar aquí & 4,22 & 0,97 & 4,5 & 0,52 & 4,4 & 0,88 \\
\hline 3. Cuando llega un nuevo profesor, recibe una inducción & 3,71 & 1,25 & 4,3 & 1,11 & 3,5 & 0,88 \\
\hline $\begin{array}{l}\text { 4. Existe confianza y buena comunicación entre todos los } \\
\text { actores de la comunidad escolar }\end{array}$ & 3,67 & 1,01 & 4,4 & 0,72 & 3,5 & 0,52 \\
\hline 5. Los padres y apoderados confían en la escuela & 4,04 & 0,79 & 4,2 & 0,66 & 4,1 & 0,78 \\
\hline 6. Existe una comunicación fluida con los padres y apoderados & 3,92 & 0,91 & 3,7 & 0,66 & 3,7 & 0,97 \\
\hline $\begin{array}{l}\text { 7. Percibimos a los estudiantes contentos y a las familias } \\
\text { satisfechas }\end{array}$ & 3,81 & 0,84 & 3,7 & 0,91 & 3,4 & 0,88 \\
\hline 8. Hay muchas tradiciones que nos hacen sentir parte de ella & 3,73 & 1,18 & 3,7 & 0,83 & 3,4 & 1,01 \\
\hline Total (promedio) & 3,86 & 0,75 & 4,1 & 0,80 & 3,6 & 0,80 \\
\hline
\end{tabular}

Fuente: elaboración propia.

Figura 3. Análisis descriptivo según opciones de respuesta de la dimensión Cultura Escolar Inclusiva para profesionales

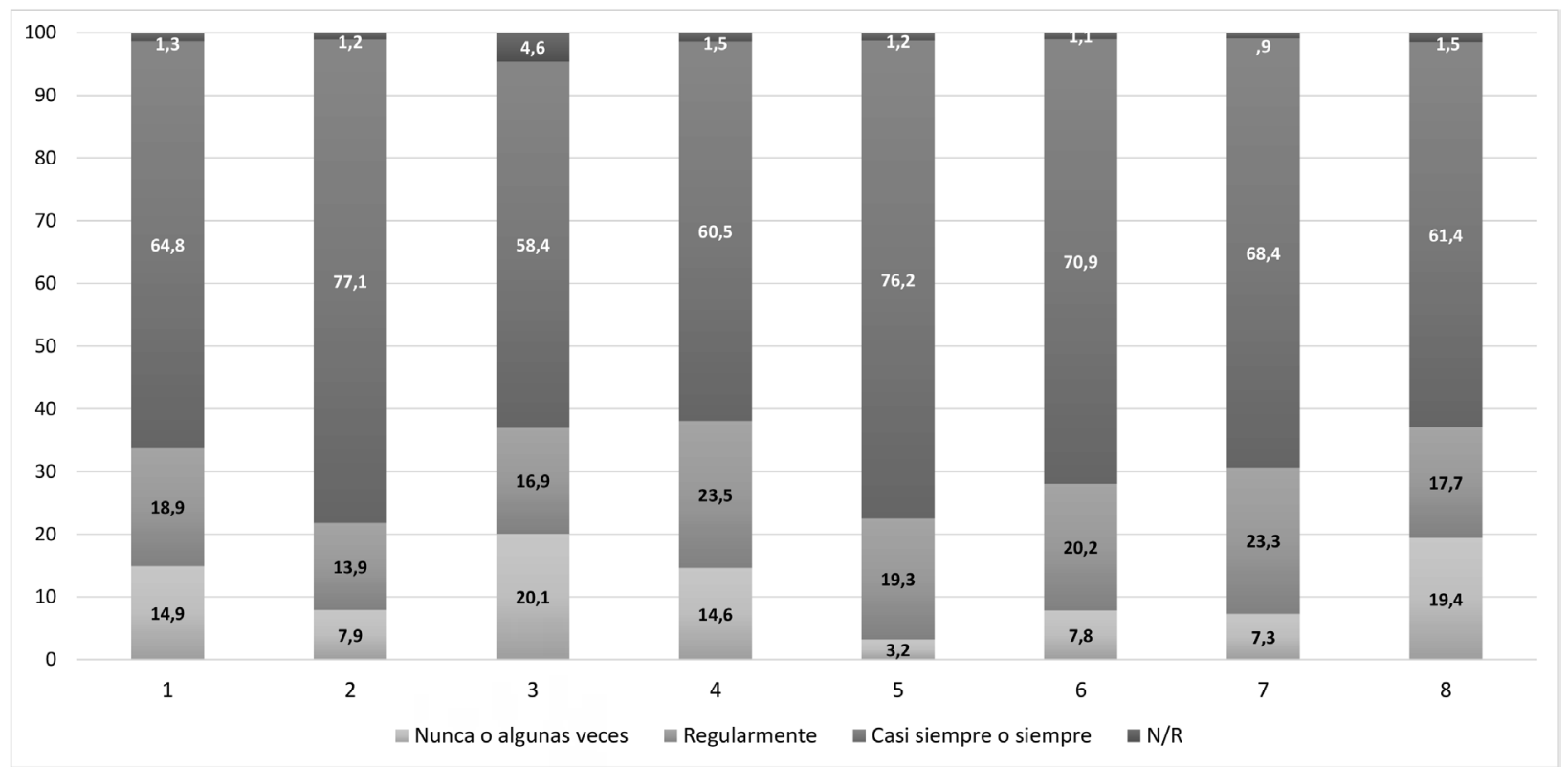

Fuente: elaboración propia. 
En sintesis, las entrevistas señalan que los adultos, profesionales y padres concuerdan en que existe una buena convivencia entre las diversas personas de la escuela y sienten a los estudiantes contentos y a las familias satisfechas. Destacan la confianza y la buena comunicación. Los estudiantes subrayan la calidad del equipo docente y los apoyos que reciben del equipo directivo y de los profesionales de apoyo.

En la escuela B, y en relación con la dimensión Convivencia Escolar Inclusiva, los estudiantes consideran que hay profesores capacitados en la escuela y que sus padres y apoderados reciben atención especial cuando lo necesitan. Mencionan que los adultos se llevan bien entre ellos y que se apoyan cuando hay problemas. No obstante, señalan que el director no siempre entrega las ayudas cuando ellos como alumnos lo necesitan: "Me gusta como son los profesores: buena onda. Además mi mamá conoce a todos los profesores, sabe cómo son, y si me cambia no sabe cómo serán los profesores [...]. Mi mamá nunca ha hablado con el director, solo con el tío J. [jefe de la Unidad Técnica Pedagógica - UTP], porque casi nunca la han llamado o nunca se encuentran en la oficina" (estudiante de $6^{\text {to }}$ básico).

Los profesionales entrevistados no concuerdan en la idea de que existe ambiente familiar dentro de la escuela. Tampoco perciben que los estudiantes estén contentos y las familias satisfechas. En el caso de los apoderados, tampoco hay consenso en que exista ambiente familiar, confianza y buena comunicación entre los diversos estamentos. Y la mayoría considera que no existen tradiciones dentro de la escuela que los hagan sentir a todos partícipes de la vida escolar: "eso para mí fue chocante: encuentro tanta falta de amabilidad en esta escuela de repente [...] la gente está muy enojada en este colegio, por ejemplo, los auxiliares, las familias, etc." (profesor, escuela B).

Los profesores entrevistados de la escuela B concuerdan en que no hay convivencia escolar acorde con lo que se espera de la escuela y señalan que no existe ambiente familiar ni confianza o buena comunicación entre adultos. Una mirada distinta tienen los estudiantes, quienes señalan que los adultos se llevan bien entre ellos, aunque explicitan que el rol directivo es menor cuando ellos tienen necesidades específicas.

En sintesis, se observó un alto nivel de coherencia y cohesión en los relatos de los participantes de la escuela $A$, quienes destacaron una convivencia escolar de tipo familiar, una muy buena acogida y apoyo de parte de profesores, y una directora presente y atenta a sus necesidades. En cambio, en la escuela B no se observó tal nivel de coherencia y cohesión entre los distintos participantes, y la escuela fue caracterizada con un clima de convivencia poco familiar. A pesar de que los profesores fueron caracterizados como presentes y atentos a sus necesidades, los estudiantes delatan la falta de presencia y posible liderazgo del director.

A continuación se presentan las categorías adicionales que emergieron del análisis de la fase cualitativa.

\section{RELACIONES INTERPERSONALES:}

En la escuela A, uno de los elementos más evidentes para quien se establece en esta escuela por un largo periodo de tiempo es la buena relación entre profesores, directivos y profesionales no docentes. Esto se visualiza principalmente entre profesores e inspectores de patio, entre las familias y la directora de la escuela y entre profesores de $1^{\text {er }} y$ $2^{\text {do }}$ ciclo. Se destacan estos tres aspectos, pues generalmente son lo que resultan más conflictivos en el ámbito escolar, los que luego veremos en detalle en la escuela B. La profesora jefe de $5^{\text {to }}$ básico nos comenta lo siguiente en un consejo de profesores: "Tengo colegas (de otras instituciones) que no llegan contentos a la escuela, que llegan a la escuela con mala cara, cansados, frustrados; en cambio, acá todos llegan a la escuela con ganas de estar con los niños, de estar con los colegas y de trabajar en un ambiente familiar. Además nos caracteriza el sentido del humor" (profesora de Educación Física). 
Lo que dice esta profesora lo notamos por primera vez en el primer consejo de profesores al que asistimos. Los profesores, irreflexivamente, nos brindaron su apoyo, no sin la correspondiente cuota de humor y de bromas. También lo notamos cuando ingresamos al aula o acompañamos a una de las inspectoras de patio en sus diversas funciones cotidianas. La directora de la escuela nos comenta que no siempre existió buen ambiente laboral entre los diversos estamentos. Hace 405 años las relaciones estaban quebradas. Con el arribo de la jefa de UTP las cosas empezaron a cambiar, pues ella necesitaba que, para transitar hacia un enfoque pedagógico, existiera primero una adecuada convivencia escolar. Una serie de prácticas relacionadas con la mejora de las relaciones humanas hicieron que la escuela dejara atrás la intemperancia y se encaminara a una escuela que, aparte de tener relaciones humanadas cohesionadas, tuviera buen rendimiento escolar.

En cambio, en la escuela B, nuestra discreta experiencia etnográfica se debió a la fragmentación de sus relaciones humanas. Como se anticipó en el párrafo anterior, esto se advirtió principalmente, en primer lugar, entre profesores e inspectores de patio, pues se cruzaban roles y funciones, sobre todo en el manejo de los problemas de conducta. Los profesores decían que los inspectores no cuidaban a los alumnos cuando eran expulsados de aula y los inspectores decían que los profesores no sabían gestionar la convivencia. Esto ha generado asperezas en las relaciones humanas, así como intolerancias respecto de los límites de cada actor educativo. En segundo lugar, entre los apoderados y el director de escuela, ya que los primeros criticaban la gestión de recursos del segundo. El centro de padres comentaba que el director se tomaba atribuciones que no correspondía con el uso de algunos fondos. Esto generó una fractura entre el centro de padres, los apoderados y la dirección. En tercer lugar, entre profesores de $1^{\text {er }}$ y $2^{\text {do }}$ ciclo, por causa de ciertas complicaciones arrastradas desde años atrás, que se materializaron, en cierto punto, pero no de manera determinante, en los rangos etarios, ya que los profesores de primer ciclo prefieren mantener ciertos ritos y costumbres de la escuela que los de segundo ciclo quieren cambiar (uso de salas, de espacios de recreación, etc.). Por otro lado, los profesores de $2^{\text {do }}$ ciclo se quejan de que los otros profesores no asisten ni apoyan las marchas y los paros. La encargada de la biblioteca, que lleva 17 años en la escuela, nos comenta lo siguiente:

Yo he valorado más a otros directores que han pasado por esta escuela. Acá hay harta división de grupos. Empezaron a llegar profesores jóvenes, y los profesores antiguos tenían sus reglas, su normativa, y los profesores jóvenes no aceptaron esas normas, no las cumplian y por lo tanto empezaron a ver (sic) estos roces, y el segundo roce comenzó en el paro, cuando no había consenso de irse o no irse a paro, y los profesores más jóvenes estaban de acuerdo con irse a paro en algunos momentos del año, no asi los profesores más antiguos, y asi se vuelve acentuar el conflicto.

La última etapa de nuestro trabajo etnográfico se complicó precisamente por estos motivos. Para profundizar en el ámbito de las relaciones interpersonales tuvimos que preguntar a diversos actores y de forma diferenciada. La escuela era reticente a exponer esta debilidad. En ese sentido, obtuvimos respuestas gratificantes en actores menos protagonistas, como la bibliotecaria y los ayudantes de aula, no así de los profesionales, como profesores y directivos.

\section{ORGANIZACIÓN DE ROLES Y FUNCIONES:}

Una de muchas formas en que se puede garantizar la precisión de roles y funciones en la escuela A es la claridad tanto en la planificación de nuestro trabajo etnográfico como en la coordinación de nuestra presencia en los diversos espacios de la escuela: sabíamos con claridad los conductos regulares, con quién hablar, hacia dónde dirigirnos, con quién conversar para saber cierta información, etc. Esto facilitó la inmersión en la escuela y permi- 
tió profundizar con mayor fluidez en las capas de la organización escolar. Como expresó la jefa de la Unidad Técnica Pedagógica (UTP): "Somos un equipo estable de profesores. En los últimos cinco años se han ido 203 y por problemas familiares. Incluso algunos han deseado volver. Además todos sabemos lo que tenemos que hacer y hacia dónde vamos". Al preguntársele por tal orientación, contestó que su deber es el de lograr aprendizajes significativos en todos los estudiantes "Esa es nuestra meta".

Todos los actores de la escuela sabían lo que tenían que hacer y también sabían por qué hacerlo. Los profesores organizaban sus aulas en función de las necesidades de los estudiantes y aplicaban una rutina de normalización²: esto pudimos visualizarlo en cursos de $1^{\text {er }}$ y $2^{\text {do }}$ ciclo básico. Los inspectores saludaban a los estudiantes por la mañana y luego atendían a las familias en sus diversas consultas. Los directivos estaban generalmente en el patio apoyando al resto del personal. Esta práctica sinérgica era consecuencia de una serie de consensos entre profesores y directivos.

Por el contrario, una de las frases más frecuentes durante nuestra estadía en la escuela B fue "somos un poco desordenados. Acostúmbrense", frase repetida por profesores, asistentes de la educación y directivos de la escuela. Esta falta de precisión en los roles y funciones se manifiesta en algunas prácticas improvisadas de gestión escolar, como, por ejemplo, en quienes asumían el liderazgo de ciertas reuniones, en coordinar la limpieza en las algunas dependencias de la escuela, en ejecutar protocolos de bienvenida para personas recién llegadas a la escuela, en la puesta en marcha de conductos regulares cuando la visita de alguna autoridad al establecimiento y en otras tareas menores, como la revisión de libros de clases o la entrega de información a los apoderados. Todos

2 La rutina de normalización consistía en pasar por la sala de clases con una caja para que los estudiantes dejen su teléfono celular, resguardar el uso del uniforme, pasar lista y chequear los materiales del día. Esto ocurría en los primeros 10 minutos de la sesión. los eventos antes mencionados fueron vistos en nuestra inmersión etnográfica, estuvieron sujetos a dudas e incertidumbres y se registraron en las siguientes notas de campo: "el inspector de la escuela también es jefe de UTP”; "la encargada de convivencia escolar es profesora del grupo diferencial y encargada de alimentación"; "el director no sabe quién es la persona encargada de revisar los libros de clases"; "la bibliotecaria dice que en la biblioteca no hacen aseo, porque los encargados de esa tarea no han recibido órdenes claras por parte del director".

Al igual que en la escuela A, la calidad de nuestra inmersión etnográfica en esta escuela también estuvo condicionada por la precisión que esta tenía en cuanto a roles y funciones. En el caso de la institución B, el trabajo etnográfico se vio dificultado. Muchas veces planificamos reuniones que no se concretaron, organizamos visitas que no se establecieron y, como quien hace un trámite que desconoce, pululamos entre diversos actores para conseguir respuestas simples. El director nos mandaba adonde el jefe de UTP y este nos enviaba con profesores, que por su parte nos enviaban con la encargada de convivencia escolar. Durante el transcurso de la etnografía, constatamos que tal desavenencia de roles no solo las sufrimos nosotros, pues en reuniones del consejo de profesores, los docentes también exponían quejas similares. En resumen, la escuela perdía mucho tiempo gastando energías en cuestiones de forma, antes que de fondo.

FOCO DE LA ESCUELA

Las energías de la escuela A estaban puestas en el aprendizaje de los estudiantes y esta motivación atraviesa tanto los discursos como las prácticas de profesores de $1^{\text {er }}$ y $2^{\text {do }}$ ciclo básico. Para alcanzar este objetivo, el equipo directivo ha potenciado talentos, ha reorganizado roles y funciones y, especialmente, ha instalado un "lenguaje común". O sea, es posible darse cuenta de que todos comparten un propósito mayor. Un profesor nos mencionó que en su escuela "nunca hay que bajar la guardia", haciendo referen- 
cia a no confiarse en los resultados, a estar monitoreando el aprendizaje de los estudiantes, a estar trabajando colaborativamente y a ofrecer también nuevas propuestas. Los docentes destacaron la confianza depositada en ellos por parte del equipo directivo y agradecieron la libertad pedagógica de que gozaban. Mencionaron: "cuando nos proponemos cambios lo logramos", atribuyéndole una importancia a la mantención de altas expectativas: "Nosotros pasamos desde un foco en convivencia, en el 2014 y 2015, pendientes de cómo se llevaban mejor, pero que hoy en día estamos $100 \%$ focalizados en lo pedagógico, en el rendimiento, en que los estudiantes aprendan, lean, escriban en los primeros niveles, y que vayan mejorando su aprendizaje a medida que va avanzando su escolaridad" (Jefa de UTP).

Un aspecto importante de la escuela $A$ es la mejora que ha tenido en los últimos años en la evaluación externa de pruebas estandarizadas. Actualmente se encuentran sobre el promedio de escuelas con igual nivel socioeconómico. Si bien es necesario priorizar que estas evaluaciones están sujetas a diversos factores y que, además, se confunde aprendizaje con rendimiento, conviene mencionar que también han tenido buenos resultados en evaluaciones internas, donde han podido visualizar mejoras significativas en el ámbito de la lectura, escritura, matemáticas y otras áreas en general. Pudimos observar tanto en la sala de clases como en los consejos de profesores una constante reflexión docente. Además, los temas de conversación cotidiana eran principalmente de índole pedagógica.

En relación con la escuela $\mathrm{B}$ y precisamente con lo mencionado anteriormente sobre la fractura en las relaciones humanas, el foco de esta escuela estaba puesto en la convivencia escolar, específicamente en el "llevarse bien". En una de las primeras conversaciones que tuvimos con el director le preguntamos sobre su estilo de liderazgo. Nos respondió que presumía de un liderazgo permisivo, pues prefiere evitar los problemas, "y esta escuela ya tiene hartos problemas en convivencia”, agregó. En nuestras ob- servaciones y conversaciones con la escuela, nos llamó la atención que nadie hablara de aprendizaje ni de rendimiento ni de prácticas de aula ni de estrategias innovadoras. Las conversaciones se enfocaban en las relaciones humanas, que los estamentos se relacionaran bien, con buen trato, atentos a la opinión de la familia, a que los inspectores de patio se llevaran bien con los profesores, y que los profesores estuvieran trabajando en buenas condiciones laborales. La encargada de Convivencia atribuía este foco al tipo de liderazgo presente en la escuela.

Tenemos que hacer que los niños aprendan. Entonces eso ha faltado. El liderazgo pedagógico es algo de lo que carece esta escuela; lamento decirlo, pero es asi. Hay otros liderazgos, liderazgos en la convivencia, de hacer comunidad, de hacer cultura, de eso, pero necesitamos un liderazgo pedagógico, un liderazgo que te diga 'vamos a desarrollar tales habilidades, vamos a tener niños cientificos, vamos a esto, [a dar] más apoyo a la profesora de ciencias que está haciendo un huerto, más capacitación más gente.

Lo que menciona la encargada de Convivencia también pudimos apreciarlo en nuestra estancia en la institución, especialmente en las reuniones del equipo directivo, donde las preocupaciones del director giraban principalmente en torno a temas administrativos y de relaciones humanas, especialmente enfocado en la mala relación entre profesores de primer y segundo ciclo. Nuestras observaciones refuerzan la idea de una fractura en las relaciones humanas y constatan la pérdida de foco de la escuela, sin un propósito pedagógico manifiesto para el aula y carente de una docencia innovadora.

\section{Discusión}

El objetivo de este estudio fue caracterizar y comprender la convivencia y la cultura desde la perspectiva de la inclusión escolar. Para ello se desarrollaron dos objetivos específicos: evaluar cuantitativamente las dimensiones de convivencia escolar y cultura inclusiva y ver en profundidad cómo operan 
ambas dimensiones en la práctica educativa, realizando una aproximación etnográfica a dos escuelas, identificadas con alto y bajo nivel de convivencia escolar y cultura inclusiva.

En cuanto a los resultados de la fase cuantitativa, del total de escuelas, los estudiantes reportan en la dimensión Convivencia Escolar Inclusiva una baja frecuencia de prácticas relacionadas con la capacidad de motivación de los profesores, el apoyo diferenciado en aula y disponibilidad del director para atender a los estudiantes. Como prácticas más frecuentes se mencionan las relacionadas con la apertura de la escuela a la atención de padres y apoderados. Según los padres, y en relación con la dimensión Cultura Inclusiva, la mayoría menciona que confían y que se sienten orgullos de la escuela donde están matriculados sus hijos y/o pupilos. En el caso de los profesionales, las acciones menos frecuentes son aquellas referidas a la presencia de un proceso de inducción, a la buena comunicación entre pares y a la existencia de tradiciones comunes para la escuela. La acción más frecuente es la vinculada al orgullo que sienten de pertenecer a la institución donde trabajan.

Si focalizamos en los dos casos extremos del estudio, como es de esperar, las dimensiones difieren entre sí, en lo que toca tanto a los estudiantes como a los padres y profesionales. Las mayores diferencias en el caso de los estudiantes se encuentran en el apoyo del director (alto en escuela $A$ y bajo en escuela B) y en dimensiones más valorativas, como la confianza y la buena comunicación (también mayormente reportados en escuela A). Este último punto es relevante, pues tiene vinculación directa con la definición de cultura inclusiva (Booth y Ainscow, 2015).

Los resultados de la aproximación cualitativa y comprensiva a las dimensiones evaluadas son coherentes con los hallazgos en la fase cuantitativa. Por un lado, y tras realizar observaciones en los espacios formales e informales de la escuela $\mathrm{A}$, encontramos que se deja entrever con relativa facilidad la buena comunicación entre adultos, algunos valores inclusivos, como la confianza, y una lógica escolar marcada por el ambiente familiar. Esto, además, se vio reforzado en las entrevistas. De la misma manera, en el caso de la escuela B, las observaciones también ponen de manifiesto las tensiones entre los adultos y los problemas de comunicación. Estas situaciones también fueron corroboradas en las entrevistas individuales. Por tanto, existe una coherencia lógica entre los resultados cuantitativos y la comprensión de estos en la fase cualitativa. Sin embargo, esta regla no aplica para los estudiantes. Si bien los estudiantes de ambas escuelas manifestaron resultados dispares en la aplicación del instrumento de convivencia escolar inclusiva, en la fase cualitativa no se encontraron grandes diferencias en relación con su percepción de la cultura y la convivencia. Ambos grupos de estudiantes consideran que hay un buen ambiente laboral y que las cosas funcionan de forma relativamente correcta.

Con respecto a los temas emergentes que surgieron de la fase cualitativa de inmersión etnográfica, la escuela A se presenta y se evidencia como una institución que cuenta con un equipo directivo estable y un discurso incluyente, basado en un enfoque de derechos. Su idea es que todos los estudiantes pueden aprender y tienen derecho a educarse, a pesar del contexto que los rodea cotidianamente. Por lo tanto, como institución adoptan un enfoque pedagógico inclusivo. La escuela $\mathrm{B}$, en cambio, posee un equipo directivo inestable, pues los integrantes se han ido rotando en los últimos años. Esta escuela cuenta con un director que tiene los intereses puestos en la mejora de las relaciones humanas y con un jefe de Unidad Técnica Pedagógica que aboga por tener docentes afectuosos y apasionados. Sucede que en esta institución hay conflictos internos de relaciones interpersonales y esto implica, entonces, que los esfuerzos estén puestos en el área relacional, lo que deja al descubierto que esta institución se encuentra en la fase de mejora de las relaciones interpersonales. 
Si relacionamos resultados cuantitativos y cualitativos, hallamos encuentros y desencuentros. Según el instrumento aplicado a los apoderados, estos son optimistas acerca del funcionamiento de sus escuelas. La mayoría responde que se sienten orgullosos de ser parte de la comunidad escolar y que confían en los profesores. Ambos datos se pueden constatar tanto en la escuela A como en la B, sobre todo la confianza en los profesores, la cual es bien avalada en las entrevistas con el centro de padres y en los diversos diálogos con los apoderados en general. Y en relación con la escala destinada a profesionales, estos mencionan que los profesores nuevos no reciben inducción y que no siempre existe confianza y buena comunicación entre colegas. El primer punto se puede visualizar en ambas escuelas: la práctica de la inducción no es recurrente en ninguna de las dos instituciones ni está entre las intenciones de los respectivos equipos directivos. Si bien la directora de la escuela A manifiesta que busca que al contratar busca buenas personas, no existe un protocolo que pueda ser considerado como proceso de inducción. En cuanto al segundo punto, claramente se percibe en la escuela B, la cual cuenta con una fractura en las relaciones humanas que afecta la confianza y la comunicación.

La escuela A posee una orientación inclusiva y tiene un foco pedagógico. Es decir, que tiene todas sus energías puestas en que todos y todas aprendan. En este caso, con aprendizaje nos referimos a la adquisición de contenidos curriculares en un ambiente de respeto y colaboración. Este último punto es destacable, pues la escuela, antes de adoptar un foco pedagógico, tuvo un foco en la convivencia escolar, en el entendió de que no es posible desarrollar aprendizajes en un ambiente con problemas de convivencia y que la convivencia es un medio para un fin, en este caso, un fin pedagógico. Al mismo tiempo, esta escuela supo que debía desarrollar una cultura de la inclusión, cultura que, en palabras de la jefa de la UTP, se relacionaba con la creación de un ambiente familiar, donde todos se sientan valorados y se apoyan entre sí. Por tanto, en esta escuela, tanto la convivencia escolar como la cultura inclusiva representan las condiciones mínimas para echar a andar una escuela inclusiva. Esta información se puede avalar en la literatura especializada (Fierro, 2013; López, 2014; Chaparro et al., 2015). En su contraparte, la escuela B aún no puede avanzar hacia un foco pedagógico, pues le falta desarrollar una cultura de la inclusión, y tampoco goza de una buena convivencia escolar. Si bien no queremos decir que ambos elementos sean predictivos, sí podemos sostener que se encarnan claramente como facilitadores de la inclusión escolar.

En función de esto último, conviene discutir el foco de ambas escuelas, en el momento en que las acompañamos. Si bien las dos instituciones tienen características organizativas y perfiles similares, reportan resultados cuantitativa y cualitativamente diferentes. Sin embargo, aunque hay prácticas concretas que nos permiten razonar la diferencia entre ambos centros escolares, pareciera ser que las escuelas de este estudio se encontraban en fases distintas (Bellei et al., 2014). Es decir, persiguen propósitos diferentes según las preocupaciones y las prioridades de la institución. Según el enfoque sociocultural de las comunidades de práctica, el contexto, la situación y la época son componentes de la práctica (Wenger, 2001) y, por tanto, reveladores de la madurez y complejidad de las acciones escolares. En consecuencia, no podemos solo mirar las acciones aisladas, sin considerar su contexto histórico y sociocultural. Según la experiencia etnográfica, la escuela A se encuentra en una etapa en que desean desarrollar correctamente los aprendizajes curriculares, con base en buenas relaciones interpersonales; por tanto, sus acciones se orientan en esa línea. En el caso de la escuela B, sus esfuerzos están puestos en la mejora de las relaciones humanas, pues es el área más débil y, en ese sentido, sus decisiones apuntan a la mejora del clima escolar.

Según los resultados reportados en esta investigación, convivencia escolar y cultura inclusiva son elementos claves para echar a andar procesos 
de mejoramiento educativo. Si la inclusión escolar es el proceso que asegura acceso, participación y aprendizaje de todos los estudiantes (Unesco, 2017), entonces podemos tomar como referente la escuela A para hilvanar una serie de argumentos que son útiles para pensar en una escuela con modelo inclusivo. En este sentido, los resultados de esta investigación concuerdan con los estudios de Moliner et al. (2016) y Escobedo, Sales y Ferrández (2012) en cuanto a la relevancia de una cultura inclusiva para el cambio escolar y la fluidez de los procesos organizativos y pedagógicos. De la misma manera, Mateus, Vallejo, Obando y Fonseca (2017) plantean que, cuando hay una cultura inclusiva, esta es fácilmente percibida por los miembros de la comunidad, como ocurre en el caso de la escuela A. Por su parte, Beneke y Cheatham (2015) y Ashikali y Groeneveld (2013) sostienen que la calidad de las relaciones interpersonales está sujeta a la puesta en práctica de una cultura inclusiva, tal cual fue sugerido en las categorías emergentes de este estudio.

En cuanto a la convivencia escolar inclusiva, los resultados de este estudio concuerdan con los de Lachat y Smith (2005) y Supovitz y Klein (2003) en cuanto a la importancia de la colaboración entre actores escolares, así como también en cuanto a la calidad de las relaciones interpersonales, lo que además es vinculante con una cultura inclusiva. De la misma forma, para desarrollar ambientes de buena convivencia escolar es indispensable que los actores compartan significados comunes respecto de lo que quieren lograr (Honig y Coburn, 2008). Este último punto se relaciona con el foco pedagógico de la escuela A y lo presente que está entre las distintas voces de la institución.

De esta manera, creemos que la convivencia escolar podría ser no solo un fin en sí mismo, sino también un medio para el desarrollo de escuelas inclusivas. En ese sentido, el abordaje de la convivencia escolar debe estar estrechamente relacionado con la instauración de culturas escolares inclusivas, pues comparten propósitos (calidad y carácter de la vida escolar, de los valores y de las relaciones interpersonales) y dimensiones (acogida, colaboración, estimulación, etc.).

La convivencia escolar, cuando es comprendida y abordada como convivencia democrática e inclusiva, permite mejorar la calidad de vida de los estudiantes en las escuelas. Esto, a su vez, refuerza el desarrollo de una cultura inclusiva. Dado que la cultura escolar se ha erigido como una dimensión clave en el desarrollo de escuelas inclusivas, sostenemos que es probable que el desarrollo de culturas escolares inclusivas facilite la sustentabilidad y sostenibilidad en el tiempo de prácticas de convivencia escolar democráticas, pacíficas e inclusivas. Esto se fortalece si además consideramos las subdimensiones que componen el constructo cultura inclusiva: a) construcción de comunidad y b) establecimiento de valores inclusivos, dentro de los cuales se encuentran valores como participación, confianza, respeto de la diversidad y justicia, elementos que también comparte la literatura sobre convivencia escolar (Fierro, 2013; López, 2014; Chaparro et al., 2015). No obstante, conviene seguir discutiendo ambos conceptos a la luz de sus semejanzas y similitudes.

En síntesis, convivencia escolar y cultura inclusiva son elementos reveladores de una escuela que avanza y mejora. Si bien la convivencia escolar ha adquirido paulatinamente fuerza en el ámbito de las políticas públicas, menos protagonista ha sido la cultura inclusiva en esa área. Creemos que, mientras las políticas educativas no hagan suyo el ideal de la inclusión con foco en el ámbito cultural, la inclusión se limitará al ámbito del acceso. Sugerimos descongestionar la escuela de las presiones externas del Sistema de Medición de la Calidad de la Educación (Simce) en cuanto a rendición de cuentas, con el propósito de que asumen de manera local y situada el ideario inclusivo. Consideramos que las políticas educativas deben apropiarse de la cultura inclusiva como una parcela de conocimiento relevante de la inclusión, intención que debe ser gestionada con el liderazgo escolar y en el ámbito de las prácticas docentes. 
Por último, como las limitaciones de este estudio están en el rango etario donde fue aplicada la escala para estudiantes ( $8^{\text {vo }}$ básico) y en el ámbito es- colar (enseñanza básica), se sugiere que para futuros estudios se profundice en otros ámbitos educativos, como el de la educación inicial y la enseñanza media.

\section{Referencias}

Agell, M., Sala, G.y Torrent, J. (2013). Participación de todo el alumnado, éxito y mejora de la escuela. Análisis de las barreras más relevantes y cómo superarlas. En C. Giné (ed.), La educación Inclusiva. De la exclusión a la plena participación de todo el alumnado (pp. 63-80). Barcelona: Cuadernos de Educación.

Anderson, S. (2010). Liderazgo directivo: Claves para una mejor escuela. Psicoperspectivas, 9(2), 34-52. Recuperado de https://dx.doi.org/10.5027/psicoperspectivas-Volg-Issue2-fulltext-127

Assaél, J., Acuña, F., Contreras, P. y Santa Cruz, E. (2018). La etnografía en el estudio de los procesos de construcción cotidiana de política educativa en espacios escolares. En Assael y Valdivia (Eds), Lo cotidiano en la escuela. 40 años de etnografía escolar en Chile (pp. 275-290). Santiago: Editorial Universitaria.

Ashikali, T. y Groeneveld, S. (2015). Diversity Management in Public Organizations and Its Effect on Employees' Affective Commitment: The Role of Transformational Leadership and the Inclusiveness of the Organizational Culture. Review of Public Personnel Administration, 35(2), 146-168. DOI: http://doi. org/10.1177/0734371X13511088

Astor, R., Benbenishty, R. y Estrada, J. (2009). School violence and theoretically typical schools: The principal's centrality in orchestrating safe schools. American Educational Research Journal, 46(2), 423-461. Recuperado de https://www.researchgate.net/publication/250185077_School_Violence_and_Theoretically_Atypical_Schools_The_Principal's_Centrality_in_Orchestrating_Safe_Schools

Bellei, C., Valenzuela, J. P., Vanni, X. y Contreras, D. (2014). Lo aprendí en la escuela. ¿Cómo se logran procesos de mejoramiento escolar? Santiago: Universidad de Chile - Unicef.

Beneke, M. y Cheatham, G. (2015). Inclusive, democratic family-professional partnerships: (Re)conceptualizing culture and language in teacher preparation. Topics in Early Childhood Special Education, 35(4) 234-244. DOI: https://doi.org/10.1177\%2Fo271121415581611

Berg, J. y Cornell, D. (2016). Authoritative school climate, aggression toward teachers, and teacher distress in middle school. School Psychology Quarterly, 31(1), 122-139. DOI: https://doi.org/10.1037/spq0000132

Berkowitz, R., Moore, H., Astor, R. y Benbenishty, R. (2017). A research synthesis of the associations between socioeconomic background, inequality, school climate, and academic achievement. Review of Educational Research, 87(2), 425-469. DOI: https://doi.org/10.3102/0034654316669821

Bilbao, M.A. (2014). Tensiones y contradicciones para el desarrollo del bienestar subjetivo en la escuela. En J. C. Oyanedel (ed.), Debates sobre el Bienestar y la Felicidad. Santiago: RIL. 
Blanco, R. (2006). Hacia una escuela para todos y con todos. Boletín del Proyecto Principal de Educación para América Latina y el Caribe, 48, 55-72. Recuperado de http://benu.edu.mx/wp-content/uploads/2015/03/ Hacia_una_escuela_para-todos.pdf

Booth, T. y Ainscow, M. (2000). Index for inclusion. Developing leaning and participation in schools (2 ed.). Manchester: CSIE.

Booth, T.y Ainscow, M. (2015). Guía para la Educación Inclusiva. Desarrollando el aprendizajey la participación en los centros escolares. Adaptación de la $3^{\text {a }}$ edición revisada del Index for Inclusion. OEI.

Bradshaw, C., Waasdorp, T., Debnam, K. y Johnson, S. (2014). Measuring school climate in high schools: a focus on safety, engagement, and the environment. Journal of School Health, 84(9), 593-604. DOI: https://doi. org/10.1111/josh.12186

Chaparro, A., Caso, J., Fierro, C.y Díaz, D. (2015). Desarrollo de un instrumento de evaluación basado en indicadores de convivencia escolar democrática, inclusiva y pacífica. Perfiles Educativos, 37(149), 20-41. Recuperado de http://www.redaly c.org/pdf/132/13239889002.pdf

Cohen, J. (2006). Social, emotional, ethical and academic education: Creating a climate for learning, participation in democracy and well-being. Harvard Educational Review, 76(2), 201-237.DOI: https://doi.org/10.17763/ haer.76.2.j44854×1524644vn

Cohen, J., McCabe, E., Michelli, N. y Pickeral, T. (2009). School climate: Research, policy, teacher education and practice. Teachers College Record, 111(1), 180-213. Retrieved from http://www.tcrecord.org/Content. asp?Contentld $=15220$

Diaz de la Rada, Á. (2013). Etnografia de la escuela más allá de la etnografía y de la escuela: tensiones disciplinares y aplicabilidad de los saberes etnográficos. Educación y Futuro, 29, 13-99.

Dorczak, R. (2013). Inclusion through the lens of school culture. En G. M. Ruaircs, E. Ottesen y R. Precey (eds.), Leadership for Inclusive Education (pp. 47-59). Rotterdam: Studies in Inclusive Education.

Eliot, M., Cornell, D., Gregory, A.y Fan, X. (2010). Supportive school climate and student willingness to seek help for bullying and threats of violence. Journal of School Psychology, 48(6), 533-553. DOI: https://doi.org/10.1016/j. jsp.2010.07.001

Escobedo, P., Sales, A. y Ferrández, M. R. (2012). La cultura escolar en el cambio hacia la escuela intercultural inclusiva. Edetania, 41, 163-175. Recuperado de https://revistas.ucv.es/index.php/Edetania/article/view/262

Fierro, C. (2013). Convivencia inclusiva y democrática: Una perspectiva para gestionar la seguridad escolar. Sinéctica, 40, 1-18. Recuperado de https://sinectica.iteso.mx/index.php/SINECTICA/article/view/47

Hirmas, C. y Eroles, D. (2008). Convivencia democrática, inclusión y cultura de paz: Lecciones desde la práctica educativa innovadora en América Latina. Santiago de Chile: Unesco. 
Honig, M. I.,y Coburn, C. (2008). Evidence-based decision making in school district central offices toward a policy and research agenda.Educational Policy,22(4),578-608.DOI:https://doi.org/10.1177\%2Fo895904807307067

Holstein, J. A. y Gubrium, J. F. (1995). The active interview. Thousand Oaks: Sage. DOI: http://dx.doi. org/10.4135/9781412986120

Lachat, M. A. y Smith, S. (2005). Practices that support data use in urban high schools. Journal of Education for Students Placed at Risk, 10, 333-349. DOI: https://doi.org/10.1207/s15327671espr1003_7

López Melero, M. (2012). La escuela inclusiva: una oportunidad para humanizarnos. Revista Interuniversitaria de Formación del Profesorado, 74(26-2), 131-160. Recuperado de http://www.redalyc.org/articulo. oa?id=27426890007

López, V. (2014). Convivencia escolar. Apuntes. Educación y Desarrollo Post-2015, 4, 1-18. Recuperado de http:// www.unesco.org/new/fileadmin/MULTIMEDIA/FIELD/Santiago/pdf/APUNTEO4-ESP.pdf

López, V. y Carrasco, C. (eds.) (2015). Nosotros si podemos: Aprendiendo a mejorar la convivencia escolar. Valparaíso: Pontificia Universidad Católica de Valparaíso y Centro de Investigación Avanzada en Educación.

López, V. y Valdés, R. (2018) Construcción y validación de instrumentos para evaluar prácticas de convivencia escolar en profesionales y padres. Actualidades Investigativas en Educación, 18(3). Recuperado de https:// revistas.ucr.ac.cr/index.php/aie/article/view/34328

López, V., Ascorra, P., Bilbao, M. de los A., Morales, M., Moya, I., Oyanedel, J. C. (2012). El ambiente escolar incide en los resultados PISA 2009: Resultados de un estudio de diseño mixto. En Mineduc (comp.), Evidencias para las politicas públicas en educación. Selección de investigaciones. Concurso extraordinario Fonide-PISA (pp. 115-136). Santiago: Mineduc. Recuperado de https://s3.amazonaws.com/archivos.agenciaeducacion.cl/ documentos-web/Estudios+Internacionales/PISA/Evidencias_para_Politicas_Publicas_en_Educacion_ FONIDE_PISA.pdf

López, V., Montecinos, C., Rodríguez, J. I., Calderón, A. y Contreras, J. F. (2011). Enacting solidarity to address peer to peer aggression in schools. En C. Sleeter y E. Soriano (eds.), Building solidarity across communities of difference in education: International Perspectives. (pp. 23-44). Nueva York: Teachers' College Press.

Mateus-Cifuentes, L. E., Vallejo-Moreno, D. M., Obando-Posada, D. y Fonseca-Durán, L. (2017). Percepción de las prácticas y cultura inclusiva en una comunidad escolar. Avances en Psicología Latinoamericana, 35(1), 177191. DOI: http://dx.doi.org/10.12804/revistas.urosario.edu.co/apl/a.4854

Mena I., Becerra S. y Castro P. (2011). Gestión de la convivencia escolar en Chile: Problemáticas, anhelos y desafíos. En J. Catalán (ed.), Psicología educacional: Proponiendo rumbos, problemáticas y aportaciones (pp. 81-112). La Serena: Editorial Universidad de La Serena.

Ministerio de Educación (2015). Marco para la buena dirección y el liderazgo escolar. Santiago: Centro de Perfeccionamiento, Experimentación e Investigaciones Pedagógicas. 
Moliner-García, O., Sales, A. y Escobedo, P. (2016). Possibilities and limits to build an institutional culture from the classroom practices shared: the case of inclusive intercultural education. Estudios sobre Educación, 30 , 51-70. http://doi.org/10.15581/004.30.51-70

Morales, M. y López, V. (2019). Políticas de convivencia escolar en América Latina: Cuatro perspectivas de comprensión y acción. Education Policy Analysis Archives, 5/6, 1-25. DOI: https://doi.org/10.14507/epaa.27.3526

Onwuegbuzie, A. y Leech, L. (2005). On becoming a pragmatic researcher: The importance of combining quantitative and qualitative research methodologies. International Journal of Social Research Methodology: Theory and Practice, 8, 375-387. DOI: https://doi.org/10.1080/13645570500402447

Páez, D. y Martín-Beristain, C. (2011). Superando la violencia colectiva y construyendo cultura de paz. Madrid: Fundamentos.

Quintana, A. (2006). Metodología de investigación científica cualitativa. En A. Quintana yW. Montgomery (eds.), Psicología: Tópicos de actualidad (pp. 47-84). Lima: Universidad Nacional Mayor de San Marcos.

Rodríguez, F.y Ossa, C. (2014). Valoración del trabajo colaborativo entre profesores de escuelas básicas de Tomé, Chile. Estudios Pedagógicos, 4O(2), 285-301. DOI: http://doi.org/10.4067/S0718-07052014000300018

Sekkel, C., Zanelatto, R. y Brandao, S. (2010). Ambientes inclusivos na educação infantil: possibilidades e impedimentos. Psicologia em Estudo, 15(1), 117-126. DOI: http://doi.org/10.1590/S1413-73722010000100013

Schildkamp, K.y Ehren, M. (2013). From "intuition" to "data"-based decision making in Dutch secondary schools? En Data-based Decision Making in Education (pp. 49-67). Holanda: Springer.

Stainback, S.y Stainback, W. (eds.) (1999). Aulas inclusivas. Madrid: Narcea.

Superintendencia de Educación (2014). Cuenta pública 2014. Santiago.

Supovitz, J. A. y Klein, V. (2003). Mapping a course for improved student learning: How innovative schools systematically use student performance data to guide improvement. Report. Philadelphia: Consortium for Policy Research in Education.

Thapa, A., Cohen, J., Guffey, S. y Higgins-D’alessandro, A. (2013). A review of school climate research. Review of Educational Research, 83(3), 357-385. Recuperado de https://doi.org/10.3102/0034654313483907

Tirado, M. y Conde, S. (2015). Relaciones entre algunas acciones preventivas sobre la convivencia escolar en centros de buenas prácticas. Estudios sobre Educación, 29, 29-59. DOI: https://doi.org/10.15581/004.29.29-59

Unesco (2017). Guía para asegurar la inclusión y la equidad en educación. Agenda Mundial de Educación 2030. París. Disponible en http://unesdoc.unesco.org/images/0025/002595/259592s.pdf

Unesco (2016). Informe de Resultados TERCE: Factores asociados. Tercer Estudio Regional Comparativo y Educativo. Santiago: Oreal/Unesco. 
Unesco (2013). El Informe Situación Educativa de América Latina y el Caribe: Hacia la educación de calidad para todos al 2015 para todos. Unesco.org. Recuperado de http://www.unesco.org/new/fileadmin/MULTIMEDIA/FIELD/Santiago/images/SITIED-espanol.pdf

Unesco (2008). Los aprendizajes de los estudiantes de América Latina y el Caribe. Primer reporte del Segundo Estudio Regional Comparativo y Educativo, SERCE. Santiago: Oreal/Unesco.

Unesco (2005). Secondary education reform: Towards a convergence of knowledge acquisition and skills development. Disponible en http://old.unesco.kz/publications/ed/secondary_educatin_reform.pdf

Unesco (1994). Declaración de Salamanca y marco para la acción. Salamanca.

Valcarce, M. (2011). De la escuela integradora a la escuela inclusiva. Innovación Educativa, 21, 119-131. Recuperado de http://hdl.handle.net/10347/6228

Valdés, R., López, V. y Chaparro, A. (2018). Convivencia escolar: adaptación y validación de instrumento mexicano en Chile. Revista Electrónica de Investigación Educativa, 20(3), 80-91. DOI: https://doi.org/10.24320/ redie.2018.20.3.1720

Vázquez, F. (1994). Análisis de contenido categorial: el análisis temático. Barcelona: Universidad Autónoma de Barcelona.

Watts, E. y Erevelles, N. (2004). These deadly times: Reconceptualizing school violence by using critical race theory and disability studies. American Educational Research Journal, 41(2), 271-299. DOI: https://doi.org/1 $0.3102 \% 2 \mathrm{FoOO} 28312 \mathrm{O} 41002271$

Wenger, E. (2001). Comunidades de práctica: aprendizaje, significado e identidad. España: Paidós. 